\title{
Deserción en estudiantes de enfermería en cuatro universidades del Perú
}

\author{
HERNAN SANABRIA \\ Universidad Nacional Mayor de San Marcos, Facultad de Medicina.
}

\begin{abstract}
RESUMEN
OBJETIVO: Determinar los factores de riesgo asociados a la interrupción de los estudios de enfermería en algunas universidades del Perú, así como determinar la magnitud de la deserción según factor de riesgo. DISEÑO: Tipo caso-control, durante 1999-2000. MATERIAL Y MÉTODOS: Se estudió los siguientes factores de riesgo: salud, económicos, personales, familiares, vocacionales, laborales, judiciales-policiales, académicos y de adaptación a la vida universitaria. La muestra estuvo conformada por 88 casos distribuídos como sigue: 24 estudiantes de las ciudades de Huacho e Iquitos y 20 de las ciudades de Lima y Trujillo. La muestra no incluyó estudiantes de la UNMSM, por no contar con la autorización correspondiente. Los controles estuvieron conformados por 65 alumnos no desertores de las mismas universidades. RESULTADOS: El factor de riesgo de deserción que tuvo la mayor fuerza de asociación fue el factor vocacional, seguido del factor económico y del factor académico, acorde al odds ratio y sus limites inferiores de intervalo de confianza $95 \%$ superiores a 1 . Un $42 \%$ de estudiantes presentó a la falta vocación como factor de riesgo de deserción, seguido del factor económico con $36 \%$. CONCLUSIONES: Existe una alta asociación entre los factores vocacional y económico, así como, una leve a moderada asociación del factor académico-rendimiento con la deserción de los estudiantes de enfermería de las universidades estudiadas.
\end{abstract}

Palabras clave: Estudiantes de enfermería; estudiantes universitarios; factores de riesgo.

\section{DESERTION OF NURSING STUDENTS IN FOUR PERUVIAN UNIVERSITIES SUMMARY}

OBJECTIVE: To determine the risk factors associated to desertion of nursing studies in some universities of Peru, as well as to determine the magnitude according to each risk factor. DESIGN: Case-control epidemiological study done during 1999-2000. MATERIAL AND METHODS: The following risk factors were studied: health, economy, personal, familial, vocational, working, police-judicial, academic and adaptation to the university life. The sample consisted of 88 cases distributed as follows: 24 students from Huacho and Iquitos and 20 from Lima and Trujillo. The sample did not include students from San Marcos University as there was no approval from the Nursing Department and the Faculty of Medicine. Control students were 65 no desertor students from the same institutions. RESULTS: Most desertion associated risk factor according to OR and its lower CI $95 \%$ higher than 1 was the vocational, followed by the economical and the academic factors. Forty two per cent of the students hadn't vocation and $36 \%$ economical problems. CONCLUSIONS: There is a high association between vocation and economy as well as a light to moderate association of low academic performance and desertion of nursing students in the universities studied.

Key words: Students, nursing; university, students; risk factors.

Correspondencia:

Dr. Hernán Sanabria Rojas

Facultad de Medicina - UNMSM.

Av. Grau 755. Lima 1, Perú

E-mail:hernan_@hotmail.com 


\section{INTRODUCCIÓN}

La educación profesional es una función que acontece de manera natural e inevitable para que las personas se formen y desarrollen sobre la base de sus mayores, en dirección a sus mismas actividades dentro de la institución universitaria. La deserción universitaria constituye un problema para la educación universitaria e incluso, para otros niveles, como la educación secundaria y primaria. Las discusiones sobre el tema incluyen pobreza, falta de cultura, prejuicios raciales, conflictos familiares y emocionales, desarraigo de la vecindad, movilidad urbana. La deserción implica una pérdida del capital de recursos humanos para la familia, la comunidad y para el propio país y conlleva sentimientos de frustración que todo ser humano tiene cuando no se logra el objetivo. Las estrategias de contrarrestar estas deserciones en los niveles no universitarios han dado resultados sólo temporales, pues al final los estudiantes siguieron desertando hasta en $75 \%\left({ }^{1}\right)$. Este porcentaje es similar al encontrado en un estudio $\left({ }^{2}\right)$ en estudiantes de enfermería de una universidad peruana, situación que fue catalogada de alarmante. En general, la deserción como fenómeno que viene siendo observado desde hace mucho tiempo, no ha sido estudiada apropiadamente. ¿Qué está pasando? ¿O es que aún no existe la suficiente motivación? ¿Se requiere hacer cambios en la currícula de estudios? O, talvez, ¿que los profesores se adapten al modo de vida de los estudiantes menos privilegiados?

En el Perú, la deserción y repitencia en la Universidad indican con claridad que, en muchos casos, la base escolar-escuela y colegio-familia ha sido débil. En otras circunstancias, el proceso de admisión no ha permitido detectar a quienes realmente servían para seguir estudios universitarios $\left({ }^{2}\right)$. En este sentido, un número significativo de alumnos no ha sabido responder a las exigencias que lo hubiera conducido a logros satisfactorios en la Universidad y posterior desempeño en bien de la sociedad. Se ha señala- do $\left({ }^{1,3}\right)$ que la causa principal de la crisis permanente que vive la educación universitaria de adultos -incluye deserción- en Latinoamérica, es la teoría pedagógica que sustenta al diseño, planificación y administración de la educación; sus efectos se reflejan en los problemas académicos, administrativos, económicos, políticos. A pesar de la importancia que se le puede atribuir al tema de la deserción universitaria, los estudios de esta naturaleza son en general escasos o inexistentes en muchas bibliotecas $\left({ }^{4}\right)$. Se ha sugerido $\left({ }^{5}\right)$ factores que podrían estar asociados a la deserción, como la insuficiente vocación y capacidad para los estudios superiores; un autor refiere que los estudiantes ingresan a la Universidad en busca de un "cartón", vale decir el título. En el área de la salud, es conocido que algunas veces las enfermedades condicionan los estudios. Así, una tesis peruana $\left({ }^{6}\right)$ encontró que la tuberculosis condicionaba, en muchos casos, la aptitud para los estudios de medicina. Igualmente, en su tesis García $\left({ }^{7}\right)$ encontró que los postulantes a estudios médicos presentaban condiciones que fueron consideradas predictores de deserción; los factores tomados en cuenta fueron estado civil de los estudiantes, propiedad de la residencia, grado de instrucción de los padres, tipo de pensión, trabajo y salud. Un informe preliminar $\left({ }^{8}\right)$ de una investigación sobre deserción hecha por estudiantes de medicina de la Universidad Nacional Mayor de San Marcos durante la práctica del curso de epidemiología, encontró que el mayor porcentaje de deserción se debió a razones de salud, económicas, vocacionales, familares, personales, desajuste al medio universitario, entre otras, de menor frecuencia. Es pues de suma importancia considerar que los estudiantes empiezan sus carreras con un nivel de motivación muy alto y, con el transcurso del tiempo, esta motivación disminuye, traduciéndose en un bajo rendimiento académico e inclusive en abandono de los estudios $\left({ }^{9}\right)$. Es posible la existencia de otros factores de riesgo de deserción -no considerados en este estudio-, como por ejemplo la personalidad (a), inseguridad en la carrera por

(a) Máximo Jorge Solano Mercado. La orientación vocacional y la personalidad. Trabajo monográfico para optar el título de Licenciado en Psicología. Universidad Nacional Federico Villarreal, Facultad de Psicología. Lima (Perú) 1994. 
falta de hábitos de estudio (b) e inclusive por consumo de bebidas alcohólicas $\left({ }^{10}\right)$, que en determinadas circunstancias podrían ocasionar problemas académicos y así una eventual deserción.

Enfocando el problema en enfermería, actualmente la carrera profesional en el Perú es de nivel universitario y tiene una duración de 5 años. La imagen de la enfermera ha venido cambiando a través de los últimos años, percibiéndose como un trabajo tecnicista y predominantemente hospitalario $\left({ }^{11}\right)$. El Instituto Nacional de Estadística e Informática del Perú (12) informó que los índices de deserción universitaria se incrementan en las carreras que permiten estudiar y trabajar, como suele suceder con las estudiantes de enfermería, trabajo social, economía; por el contrario, la currícula de las escuelas de medicina exigen dedicación exclusiva, habiéndose observado una reducida deserción en este grupo estudiantil $\left(^{8}\right)$. Se ha demostrado $\left({ }^{13}\right)$ que hay una incidencia de los reglamentos académicos en la deserción y no promoción académica de los estudiantes, particularmente en las mujeres, que son la mayoría dentro de la profesión de enfermería.

Vásquez $\left({ }^{14}\right)$ realizó una investigación sobre factores biológicos, socioeconómicos y culturales que influyen en la deserción en estudiantes de enfermería de la Universidad Nacional de Trujillo. De otro lado, el medio familiar puede jugar un rol importante para que el estudiante termine su carrera o la abandone; pero, también puede ser el medio universitario mismo. Igualmente, un hogar pobre donde el nivel educativo de los padres es bajo, tendría poca influencia para que el estudiante se mantenga en sus estudios $\left({ }^{15}\right)$. Este fenómeno es preocupante en estudiantes de las ciencias de la salud, particularmente en facultades o escuelas de enfermería del Perú. Una observación personal durante el dictado del curso de epidemiología -segundo año- de la Escuela Académico Profesional de Enfermería de la Universidad Nacional Mayor de San Marcos, en 1997, nos permitió reconocer el problema de la deserción. Si bien la deserción correspondió a una deserción temprana -antes de la mitad de la carrera-, el problema parece presentarse en cualquier momento de la carrera.

Son escasos los estudios encontrados sobre la magnitud de la deserción en estudiantes universitarios, en general, y de enfermería en particular $\left({ }^{2}\right)$; igualmente es poco lo que se conoce de sus factores causales. Específicamente, se carece de estudios realizados en estudiantes de enfermería que asocien factores de riesgo a la deserción en el Perú. En este sentido, se planteó el siguiente problema de investigación: ¿Cuáles son los factores de riesgo asociados a la deserción de estudiantes de escuelas o facultades de enfermería en las universidades de Perú?. Se consideró como hipótesis, la existencia de factores de riesgo para la deserción de estudiantes de enfermería en el Perú. Sobre la base de la información previa, se planteó la existencia de factores de riesgo de deserción en estudiantes de enfermería específicos, tales como problemas de salud, económicos, personales, familiares, vocacionales, laborales, judicialespoliciales, académicos y de adaptación a la vida universitaria.

Los objetivos del estudio fueron determinar los factores de riesgo asociados a la interrupción de los estudios de enfermería en universidades del Perú. Y determinar la magnitud de la deserción según factor de riesgo estudiado.

Se definió deserción a la interrupción de los estudios de enfermería en uno de los semestres de su formación académica de 5 años, independientemente de si fue de carácter temporal o permanente.

El propósito de la investigación fue propiciar intervenciones educativas que permitan reducir la incidencia de la deserción en dicha carrera en el Perú tomando como base los resultados encontrados.

(b) Meryta Béryl Gutiérrez Briones. Dos casos clínicos incompletos: Trastornos de la conducta por déficit de atención y orientación vocacional. Trabajo monográfico para optar el título de Licenciado en Psicología. Universidad Nacional Federico Villarreal, Facultad de Psicología. Lima (Perú) 1997. 


\section{MATERIAL Y MÉTODOS}

Se realizó un estudio de tipo caso-control $\left({ }^{16}\right)$ en estudiantes de enfermería de nivel universitario, en 4 universidades del Perú, situadas en las ciudades de Lima, Huacho, Trujillo e Iquitos, entre abril de 1999 y marzo del año 2000. Los 88 casos de la muestra fueron distribuidos como sigue: 24 estudiantes de las ciudades de Huacho e Iquitos y 20 de las ciudades de Lima y Trujillo. Se consideró los siguientes factores de riesgo de deserción: salud, economía, personal, familiar, vocacional, laboral, judicial-policial, académico, adaptación a la vida universitaria y otros no especificados. Los casos de deserción fueron comparados con controles que nunca interrumpieron sus estudios. Los controles fueron similares a los casos en las siguientes variables: estudiantes de enfermería, sexo, edad promedio y universidad.

El tamaño de la muestra estuvo dentro del número calculado inicialmente para la Universidad Nacional Mayor de San Marcos UNMSM, cuya proporción de desertores en estudiantes de medicina sirvió de referencia. Los casos y los controles fueron 88 y 65 estudiantes de enfermería, respectivamente. La muestra provino de universidades nacionales del interior del país, con escuelas de enfermería con al menos 5 años de antigüedad, y una de la ciudad de Lima. Todas, excepto una, fueron universidades nacionales, situadas en las ciudades de Trujillo, Iquitos y Huacho. Inicialmente se consideró la Universidad Nacional Mayor de San Marcos en Lima; sin embargo, no se obtuvo la autorización escrita para la toma de información de sus estudiantes en su oportunidad, razón por la cual la muestra correspondió a estudiantes de enfermería de otra universidad situada también en Lima, pero de régimen privado. Cabe mencionar que las 4 universidades se encontraban registradas en la Asamblea Nacional de Rectores, máxima instancia de la Universidad Peruana.

Para la toma de datos se tuvo como referencia las listas actualizadas del alumnado matri- culado de 1994 a 1998, inclusive. Las listas permitieron identificar con relativa facilidad a los alumnos que interrumpieron sus estudios, independientemente de que la interrupción fuera temporal o definitiva. Para la inclusión de los casos y controles, se consideró deserción a la interrupción de los estudios de enfermería por espacio de 2 ó más semestres consecutivos. La separación de matrícula fue considerada como deserción. Se excluyó a los alumnos "repitentes" una vez conocida su condición. Los controles fueron seleccionados de acuerdo al sexo, edad con un rango no mayor a 2 años de diferencia y procedencia de la misma universidad; a excepción de 2 alumnos, los controles pertenecieron a la misma promoción que los casos. Se hizo una encuesta estructurada con preguntas y respuestas objetivas dirigidas específicamente a averiguar los factores de la deserción, mediante entrevista personal. Cuando los casos no fueron ubicados dentro del campus universitario, se los buscó en sus domicilios y eventualmente en sus centros laborales.

Los datos fueron procesados mediante el sistema automatizado, utilizando el Programa Epi Info versión 6, editado por el Centers for Diseases Control and Prevention de los Estados Unidos de Norteamérica. La medición del riesgo se hizo mediante el estimador odds ratio (OR). El valor de OR igual a 1 no implica riesgo, los valores mayores a 1 sí implican riesgo, siendo el riesgo alto cuando es mayor al valor de 2,5 y será más alto cuanto más se aleje de este valor o de la unidad. Para cada factor se consideró una $p$ menor de 0,05 como estadísticamente significativo, utilizándose el chi cuadrado y la prueba exacta de Fisher cuando las frecuencias fueron menores a 5; en estos casos se hizo los cálculos de intervalos de confianza al $95 \%$. El OR busca relacionar en términos de fuerza de asociación cada uno de los factores de riesgo estudiados con la deserción estudiantil; el OR como estimador del riesgo se utiliza cuando se tiene el efecto (deserción estudiantil) y se busca sus factores de riesgo. Finalmente, la magnitud fue medida en términos absolutos, así como en términos de diferencia de 
las frecuencias porcentuales entre casos y controles. Se consideró diferencia relevante a los valores mayores a $15 \%$.

\section{RESULTADOS}

Del total de 88 casos estudiados, hubo 82 mujeres y 4 hombres, siendo 22 años el promedio de edad al momento de la deserción. El 86\% de los casos desertó antes de la mitad de la carrera de 5 años, observándose que la mayoría desertó en el primer año de estudio. El factor de riesgo de deserción que tuvo la mayor fuerza de asociación fue la falta de una verdadera vocación por la profesión de enfermería -vocacional-, con un OR de 46 y un límite inferior de 6,46 para un intervalo de confianza -IC- del 95\%. El factor económico tuvo un OR de 36, seguido del factor académico con un OR de 11,2; en ambos casos, los limites inferiores del IC $95 \%$ fueron superiores a 1 ; sin embargo, el factor académico tuvo un límite inferior de 1,47. La fuerza de asociación para salud y adaptación tuvieron un OR de 4,8 y 4,6, respectivamente; sin embargo, los valores de $\mathrm{p}$ fueron mayores a 0,05 . Se hizo los cálculos del intervalo de confianza correspondientes a los factores salud y de adaptación, encontrándose el límite inferior menor a 1. Así, en el caso del factor adaptación, el IC $95 \%$ estuvo entre 0,54 y 218 . Los factores familiares y por trabajo no estuvieron asociados a la deserción estudiantil, con un OR igual a 1. En la Tabla 1 se presenta todos los factores de riesgo de deserción con sus respectivos odds ratio.

En cuanto a la magnitud, los motivos vocacionales fueron los más frecuentes, con un $42 \%$ entre el total de desertores. La mayor diferencia porcentual entre casos y controles se presentó en quienes abandonaron la carrera debido a factores vocacionales (41\%) y económicos (36\%). Una menor diferencia de porcentajes entre casos y controles se observó en quienes abandonaron los estudios debido a motivos académicos, con $16 \%$ aproximadamente. Con relación a este factor académico, hubo un total de
Tabla 1.- Medida de factores de deserción estudiantil.

\begin{tabular}{lllllll}
\hline \multicolumn{1}{c}{$\begin{array}{c}\text { Factores } \\
\text { estudiados }\end{array}$} & \multicolumn{2}{c}{ Casos } & & \multicolumn{2}{c}{ Controles } & \multirow{2}{*}{ OR } \\
\cline { 2 - 3 } & Sí & No & & Sí & No & \\
\hline Vocacional* & 37 & 51 & & 01 & 64 & 46,0 \\
Económico* & 32 & 56 & & 01 & 64 & 36,0 \\
Académico* & 13 & 75 & & 01 & 65 & 11,2 \\
Personal (embarazo) & 08 & 82 & & 01 & 64 & 6,2 \\
Salud & 06 & 82 & & 02 & 63 & 4,8 \\
Adaptación & 06 & 82 & & 01 & 64 & 4,6 \\
Familiar & 07 & 81 & & 05 & 58 & 1,0 \\
Trabajo & 01 & 87 & & 03 & 62 & 0,2 \\
\hline
\end{tabular}

(*) $p<0,05$

$20(22,7 \%)$ alumnos que repitieron el año, de los cuales $15,9 \%$ repitió un año, 4,5\% 2 años y $2,3 \% 3$ años. En el grupo control, $30 \%$ repitió un año. La diferencia porcentual por razones personales fue menor de $10 \%$, al igual que los otros factores estudiados. Con relación al factor personal, hubo 6 mujeres que abandonaron los estudios por quedar embarazadas.

Entre los factores considerados como "otros", el abandono fue mayoritariamente por traslados internos a otras carreras profesionales de la salud: medicina (06) y odontología (03). Sólo hubo 2 estudiantes que abandonaron la carrera para seguir estudios no relacionados a la salud, tales como contabilidad (01) y secretariado (01). Los problemas de adaptación a la vida universitaria se debieron principalmente a dificultades en el horario. Todos los estudiantes que siguieron otra carrera también habían respondido como motivo de su deserción al factor vocacional, por lo que no fue tabulado. En cuanto a la magnitud del factor salud, hubo 6 personas que desertaron por enfermedad, siendo la tuberculosis la patología más frecuente (04), seguido de anemia (01) y asma bronquial (01); en los controles también hubo 01 caso de tuberculosis pulmonar entre otras enfermedades, básicamente agudas -infecciones de la vía urinaria o respiratorias-. 
Finalmente, de los 7 casos que abandonaron por razones familiares, todos excepto uno, lo hicieron por enfermedad de los padres o hermanos. En la Tabla 2 se presenta detalles de las diferencias porcentuales entre casos y controles.

Tabla 2.- Magnitud:

Diferencia porcentual de factores.

\begin{tabular}{lccc}
\hline \multicolumn{1}{c}{$\begin{array}{c}\text { Factores } \\
\text { estudiados }\end{array}$} & $\begin{array}{c}\text { Casos } \\
(\%)\end{array}$ & $\begin{array}{c}\text { Controles } \\
(\%)\end{array}$ & $\begin{array}{c}\text { Diferencia } \\
(\%)\end{array}$ \\
\hline Vocacional* $^{*}$ & 42,0 & 1,5 & $+40,5$ \\
Económico* & 36,4 & 1,5 & $+34,9$ \\
Académico* & 17,3 & 1,5 & $+15,8$ \\
Personal (embarazo) & 9,8 & 1,5 & $+8,3$ \\
Adaptación & 6,8 & 1,5 & $+5,3$ \\
Salud & 7,3 & 3,1 & $+4,1$ \\
Familiar & 8,6 & 7,7 & $+0,9$ \\
Trabajo & 1,1 & 4,6 & $-3,5$ \\
\hline
\end{tabular}

* Diferencias relevantes.

No se reportó alteraciones de la personalidad. Sin embargo, hubo dos personas con aparentes fobias; una estudiante refirió haber abandonado los estudios por fobia a la sangre durante las prácticas con animales y otra que hizo lo propio al ingresar al hospital. Ningún estudiante abandonó sus estudios por razones judiciales o policiales.

\section{DISCUSIÓN}

Nuestro hallazgo más importante, dada una mayor fuerza de asociación con la deserción de estudiantes de enfermería es, sin duda, el factor vocacional. Tanto el estimador del riesgo OR, su correspondiente IC $95 \%$ y el valor $p<0,05$ apoyan la relación entre la falta de vocación por la carrera de enfermería y la deserción. En este sentido, nuestro estudio encaja dentro de un estudio cubano $\left({ }^{17}\right)$ que concluyó que la vocación es la causa esencial de la motivación para que los estudiantes eligieran la carrera de enfermería. Los resultados del presente estudio probablemente reflejen, en parte, la situación sobre la orientación vocacional en el Perú. A nuestro juicio, esta orientación estaría limitada -en los casos que se oferta- a brindar exposiciones sobre las carreras, muchas veces vacías y cansadoras y poco o nada motivadoras. Recordemos que la orientación vocacional procura que el sujeto ejerza aquella profesión para la que presenta mayor aptitud $\left.{ }^{3}\right)$. Este concepto pone de manifiesto la necesidad de hacer una medición de la aptitud de las personas para determinar sus características vocacionales, antes de la postulación a la universidad. Vásquez $\left({ }^{14}\right)$, investigando en estudiantes de enfermería de la Universidad $\mathrm{Na}$ cional de Trujillo, reveló que la buena orientación vocacional influye reduciendo la deserción significativamente. Se desconoce la situación con relación a la orientación vocacional en los casos y los controles de nuestra investigación, dado que no fue un objetivo del estudio. Al respecto, un investigador cubano $\left({ }^{18}\right)$ reveló que los estudiantes de enfermería que participaron en programas de formación vocacional y profesional fueron más eficientes que aquellos que no participaron. El factor vocacional asociado a la deserción estudiantil en enfermería trasciende esta profesión. Así, Choquehuanca $\left(^{5}\right)$ analiza los intereses vocacionales con relación a ciertos valores interpersonales entre estudiantes de diferente nivel socioeconómico. Este autor encuentra en general una carencia de vocación para los estudios superiores, los cuales muy pronto son abandonados por los estudiantes, al no poder satisfacer las mínimas exigencias. Es razonable que si el estudiante -de cualquier profesión- descubre tardíamente que sus habilidades no concuerdan con las actividades que tendrá que realizar como profesional, opta por abandonar la carrera. En otros casos, el estudiante probablemente se resigne a soportar el reto de situaciones negativas y frustrantes cuando tiene verdadera vocación por la profesión. El problema vocacional universitario en el Perú tiene su génesis en la educación secundaria. Gonzales $\left({ }^{19}\right)$ ha comprobado que desde la década de los 70s no existe un personal especializado capaz de llevar 
a cabo un programa de orientación profesional de manera cabal en colegios secundarios de la provincia de Lima, Perú. Al respecto, Rupay (c) ha señalado la importancia de la orientación vocacional como un proceso pedagógico longitudinal en la educación de nivel secundaria. Si tuviésemos que realizar intervenciones para tratar de disminuir el riesgo de deserción estudiantil, éstas deberían priorizarse en el factor vocacional, dada la fuerza de asociación y la magnitud del factor vocacional.

Por la fuerza de asociación entre el factor vocacional y la deserción en la población estudiada, se puede lanzar nuevas hipótesis de trabajo. Por ejemplo, la existencia de una orientación vocacional insuficiente está asociada a una mayor deserción de estudiantes en enfermería en el Perú, o bien, cómo inciden los reglamentos académicos en la deserción y no promoción académica de las estudiantes; o también, cómo repercute el rendimiento académico en la deserción del estudiante de enfermería. Con relación a este último, hay estudios en estudiantes mujeres de la Facultad de Ciencias de la Educación de la UNAN que demuestran su incidencia $\left({ }^{13}\right)$ en la deserción. Como consecuencia de los nuestros hallazgos, sería pertinente saber qué calidad y cuánto de orientación vocacional recibió el estudiantado de enfermería que abandonó su carrera por falta de vocación; sus resultados pueden servir para declarar después nuevas hipótesis de estudio. En la orientación profesional y ocupacional se debe considerar la vocación, los intereses y las aptitudes como elementos objetivos de la misma (d). El tema de la orientación vocacional es tratado por la psicología educacional, considerando que en estos tiempos elegir una profesión resulta compleja y difícil frente a la multiplicidad de opciones profesionales.
Otro de los factores con fuerza de asociación muy alta es el económico; igualmente está avalado por sus valores estadísticos significativos. Es razonable la asociación si tenemos en cuenta las dificultades para responder a necesidades que toda persona debe satisfacer en estos tiempos modernos de globalización. Actualmente, las universidades públicas del Perú, al igual que las privadas, tienen facultades para cobrar diversos rubros al momento de la matrícula. Se ha señalado $\left({ }^{20}\right)$ que esta privatización "silenciosa" debido a la modernidad puede eventualmente impactar en la deserción universitaria. En otras situaciones, el factor económico puede ser el generador de la "no elección" de profesiones acorde a sus condiciones socioeconómicas; o bien, el generador de la elección de profesiones que ofrecen mayores perspectivas económicas $\left({ }^{19}\right)$, pero con mayor inversión. Esta última situación, digamos de una "mala elección", demanda un gasto que eventualmente el estudiante o su familia no pueden hacerlo, propiciando necesariamente la deserción si no se encuentran los fondos respectivos en el camino. Varela $\left({ }^{21}\right)$ menciona que lo más común es elegir la carrera que más se adapta a la economía y al interés personal, atribuyendo luego explicaciones vocacionales. Un estudio $\left({ }^{14}\right)$ en la Universidad Nacional de Trujillo encontró que los bajos ingresos en las familias de los estudiantes de enfermería promueven la deserción y que un 55\% del grupo con bajos ingresos económicos abandonó los estudios. Nuestro estudio no contempló dicha información. En general, la escasez de recursos económicos y financieros constituyen uno de los problemas básicos de la educación en el Perú desde hace varias décadas, determinando, entre otras consecuencias, la deserción (e).

(c) Cecilia Rupay Velásquez. La orientación vocacional. Importancia y significado en la Escuela. Monografía. Universidad Nacional de Educación “Enrique Guzman y Valle”, Facultad de Humanidades. Chosica (Perú) 1997.

(d) Leyla Zubiaurr Dias. Evaluación de las aptitudes vocacionales. Universidad Nacional Federico Villarreal. Monografía para optar el título de Licenciada en Psicología.

(e) Valerio Palacios Flores. Recursos económicos y financieros de la Educación Peruana (DL 19326). Monografía para optar el grado de Bachiller en Ciencias de la Educación. Universidad Nacional de Educación "Enrique Guzman y Valle", Facultad de Humanidades. Chosica (Perú) 1979. 
Teniendo en cuenta esta escasez de recursos económicos, se requiere un programa de orientación vocacional bien planificado, que contemple el problema económico. Está demostrado que la buena orientación vocacional eleva la formación multidimensional del estudiante y evita de alguna manera la deserción universitaria $\left({ }^{22}\right)$. Las intervenciones focalizadas hacia el factor económico pueden ser dificultosas: sin embargo, las instituciones involucradas -Estado y universidad fundamentalmente-, deben reconocer que el factor económico es relevante, tanto por la fuerza de asociación encontrada como también por su magnitud encontrados en nuestro estudio.

Los otros dos factores asociados a la deserción en enfermería están relacionados con la propia universidad. En orden -según la fuerza de asociación-, primero el rendimiento académico y luego la adaptación a la vida universitaria. En nuestro estudio, si sólo tenemos en cuenta los valores del OR, sin tener en cuenta las otras mediciones estadísticas, podemos llegar a demostrar asociaciones falsas con la deserción estudiantil. En el caso del factor académico, fundamentalmente relacionado al rendimiento, el grado de asociación sería de leve a moderado, considerando el límite inferior de su correspondiente IC $95 \%$. Es muy probable que estas situaciones estén relacionadas con otros factores, como el económico, familiar, laboral, personalidad, estrés, etc. Es también probable que el factor de desadaptación a la vida universitaria conlleve al alumno a algún grado de frustración, en tanto permanezca en el claustro universitario. Ambas situaciones podrían terminar finalmente en deserción estudiantil. No se ha encontrado estudios que relacionen directamente a estos factores "universitarios" con la deserción en general y particularmente en enfermería. Es factible que haya otros factores colaterales, como el socioeconómico, que influyen en el rendimiento de los estudiantes de enfermería, como fuera observado por Minchola $\left({ }^{23}\right)$. En este contexto, Tipismana $\left({ }^{24}\right)$ encontró en estudiantes de enfermería con prácticas clínicas, que existe una relación estadísticamente significativa entre el estrés y el rendimiento académico. Esto podría significar que el estrés podría ser un factor de riesgo de la deserción, al influir desfavorablemente sobre el rendimiento académico. Una investigación $\left({ }^{25}\right)$ en estudiantes de odontología encontró, como explicaciones del fracaso académico, a la escasa disponibilidad de tiempo, falta de esfuerzo para el estudio y estados de tensión o estrés. Lo señalado invita a plantear nuevas hipótesis con otros factores de riesgo de deserción, como el estrés.

Dado que el IC $95 \%$ del factor adaptación tuvo su límite inferior por debajo de 1 , no se puede aceptar su asociación con la deserción de los estudiantes de enfermería en este estudio; esto es válido para todos los factores con intervalos de confianza similares y valores $p$ $>0,05$. Si hubiese que hacer intervenciones para disminuir el riesgo de deserción debido a estos factores relacionados con la universidad, éstas se orientarían principalmente al factor académico -antes que al de adaptación-, dada su mayor magnitud. De haberse tomado como un solo factor de deserción al aspecto " universitario" y no dividido en lo académico y de adaptación a la vida universitaria, es posible que hubiésemos encontrado una asociación espuria con la deserción.

El factor personal embarazo también mostró una fuerza de asociación alta; sin embargo, ésta no fue corroborada estadísticamente. Esto no es llamativo, si se toma en cuenta que esta realidad femenina necesariamente implica la dedicación de un tiempo ad hoc para su propio cuidado. Huelgan nuestros comentarios al respecto. Sin embargo, se puede especular en sus causas. Si bien es cierto que el embarazo es una realidad con muchas aristas, es menester que se tome cartas en el asunto por parte de las autoridades. Las universidades cuentan con estructuras, en muchos casos direcciones, de Bienestar Estudiantil que están en la capacidad de hacer intervenciones orientadas a que las mujeres tengan todos los elementos de juicio para posponer el embarazo mientras se está en la Universidad. Aún cuando el embarazo haya sido deseado - 
considerada como una conducta de la estudiante orientada a la satisfacción de una necesidad fisiológica dentro de su proyecto de vida-, se hace necesario dar el apoyo psicológico a las estudiantes de enfermería, tal como sucede en otras latitudes $\left({ }^{26}\right)$. Al respecto, hay estudios que demuestran la relación entre deserción con presencia de hijos en estudiantes de enfermería $\left({ }_{14}\right)$.

Un estudio $\left({ }^{27}\right)$ en estudiantes de varias profesiones en la Universidad Nacional Mayor de San Marcos sobre el rendimiento, encontró que el estudiante de bajo rendimiento académico tiende a no asumir la responsabilidad que le compete en cuanto al origen de su frustración. Igualmente, estos estudiantes no se inclinan a buscar soluciones conciliatorias. De suyo, creemos que estos factores de riesgo tienen interrelación con otros factores -no considerados en el presente estudio-, como podría ser la personalidad, los hábitos de estudio, entre otros (se ha demostrado que a mejores hábitos de estudio hay un mayor rendimiento académico), y la currícula o los reglamentos académicos $\left({ }^{13}\right)$. Finalmente, cabe mencionar que el estudio de Vásquez $\left({ }^{14}\right)$ encontró que la condición económica no es una condición sine qua non para que el rendimiento académico sea bajo.

En cuanto a salud, Aronés $\left({ }^{8}\right)$, haciendo un estudio en alumnos de la Facultad de Medicina de la Universidad Nacional Mayor de San Marcos, encontró que el mayor porcentaje de deserción se debió a salud. En nuestra investigación, el factor salud prácticamente no constituyó riesgo alguno para el abandono de los estudios. Al respecto, se ha señalado una alta prevalencia de depresión en estudiantes de enfermería $\left({ }^{28}\right)$, generalmente interrelacionados con problemas económicos y familiares. En un estudio realizado en estudiantes de medicina de la Universidad Nacional "Pedro Ruiz Gallo" de Lambayeque, Perú, se encontró una prevalencia de $16 \%$ de bebedores excesivos y patológicos entre el total de bebedores. Otro de los problemas de salud relevantes en el ambiente universitario es la tuberculosis, situación que se viene dando desde muchas décadas atrás $\left({ }^{6,29}\right)$ e inclusive, desde que son postulantes a la universidad $\left({ }^{7}\right)$. Las enfermedades, muchas de ellas engendradas antes del ingreso al recinto universitario, pueden convertirse en factores de riesgo para una deserción de los estudios universitarios $\left(^{6}\right)$.

En "otros factores", se puede considerar el estado civil, escolaridad del padre, ciertas deficiencias pedagógicas del docente, dificultad de los cursos, así como estudiar y trabajar a la vez. Estos factores fueron encontrados en estudiantes de Ingeniería Mecánica de la Universidad Nacional de Trujillo ${ }^{(30}$ ), pero no en nuestro estudio. Debe tenerse en cuenta que los estudiantes que optaron por un cambio de carrera -traslado interno para seguir otras profesiones- fueron considerados dentro del grupo del factor de riesgo vocacional, razón por la que no se hizo la medición con el estimador OR. Estos "otros" factores así como todos aquellos que tienen una magnitud menor al $15 \%$ en la diferencia porcentual entre casos y controles, deberían tener una segunda priorización en un plan de intervención para disminuir el impacto de deserción de estudiantes de enfermería en el Perú, acorde a cada realidad universitaria.

Sobre vocación, hay muchas hipótesis para estudiar en este campo. En el caso peruano, pudiera estar enmascarado con otros factores, como bien ya se ha explicado. Y muy ligado a este factor vocacional eminentemente subjetivo, está la motivación de los estudiantes de enfermería y, en general, de todos los estudiantes universitarios, para continuar estudiando. Al respecto, Lens ${ }^{(31}$ ) ha lanzado desde Bélgica una pregunta que debería ser respondida en nuestro medio también: ¿cómo la orientación hacia el futuro de los estudiantes afecta su motivación hacia la meta? En el Perú, el proceso de admisión no ha permitido detectar a quienes realmente servían para seguir estudios universitario, lo que trae problemas de deserción, pero también de rendimiento académico $\left({ }^{2,32}\right)$. Para el interés de los estudiantes de enfermería, la meta es simplemente llegar a obtener su licenciatura, razón por la cual ingresaron a la universidad en un momento dado. 
Se concluye que existe una alta asociación de los factores vocacional y económico, asi como una leve a moderada asociación del factor académico-rendimiento con la deserción de los estudiantes de enfermería de cuatro universidades del Perú. La mayor magnitud de los casos de deserción fue también debido al factor vocacional, seguido del factor económico. Estas conclusiones permiten recomendar a las instituciones y autoridades correspondientes la ejecución de intervenciones orientadas al factor vocación de una carrera profesional que incluya también sus aspectos conexos.

\section{BIBLIOGRAFÍA}

1. Goodman P. La des-educación obligatoria. Capítulo 1: La trampa universal. Barcelona: Editorial Fontanella. 1973; $181 \mathrm{pp}$.

2. Musayon Y. Relación entre el ingreso y el rendimiento académico de las alumnas de enfermería entre 1994-1997 en una universidad peruana. Rev Universidades 2001; 22: $17-21$.

3. Díaz S. Andragogía y teoría sinérgica. Ponencia en la XIII Conferencia Internacional de Andragogia. Federación Interamericana de Educación en Adultos. Costa Rica, agosto 1994.

4. Miclas T. Diagnóstico y perspectiva de la educación superior en México. Ciudad de México DF: Edit. Universidad Autónoma de México e Instituto de Investigaciones Legislativas de la Cámara de Diputados, 1993.

5. Choquehuanca M. Análisis de los intereses vocacionales en relación a ciertos valores interpersonales entre estudiantes de diferente nivel socioeconómico. Tesis de Bachiller, Programa de Filosofía, Psicología y Arte, Universidad Nacional Mayor de San Marcos. Lima (Perú), 1976.

6. Pastor M. La tuberculosis pulmonar entre los estudiantes de medicina. Tesis de Bachiller en Medicina, Universidad Nacional Mayor de San Marcos. Lima (Perú), 1957.

7. García A. La salud de los postulantes a estudios médicos. Tesis de Bachiller en Medicina, Universidad Nacional Mayor de San Marcos. Lima (Perú), 1957.

8. Aronés E, Garnique M, Gonzales I, Zavaleta J. Principales factores de deserción en estudiantes de medicina humana ingresantes 1991-1998 de la Universidad Nacional Mayor de San Marcos. V Jornadas Sanfernandinas de lnvestigación, Universidad Nacional Mayor de San Marcos, Lima (Perú), setiembre-octubre 1998.

9. Cateriano EM, Skrabonja M. Estudio del comportamiento motivacional del estudiante villarrealino. Universidad Nacional Federico Villarreal. Consejo de Investigación Científica. Trabajo de Investigación 93. Lima (Perú), 1988.
10. Valdemar M. Prevalencia del consumo de bebidas alcohólicas en estudiantes de medicina. Tesis de Bachiller en Medicina. Universidad Nacional de Trujillo. Facultad de Ciencias Médicas. Trujillo, 1987.

11. Moreno A, Murillo M. Imagen actual de Enfermería según características sociológicas de su ejercicio profesional -Trujillo, 1992. Tesis para optar grado de Bachiller en Enfermería. Universidad Nacional de Trujillo. Trujillo (Perú), 1992.

12. Instituto Nacional de Estadística en Informática. ler Censo Nacional Interuniversitario. Lima (Perú), 1996.

13. López ME. Incidencia de los reglamentos académicos en la deserción y no promoción académica de las estudiantes (mujeres) de la Facultad de Ciencias de la Educación de la UNAN en el período 1986-1991. OPS 1993; 136 pp.

14. Vásquez C. Algunos factores biológicos, socioeconómicos y culturales influyentes en la deserción de los estudiantes de enfermería. Tesis de Maestría. Universidad Nacional de la Libertad, Facultad de Educación, Escuela de Postgrado, Trujillo (Perú), 1993.

15. Romero M. La deserción estudiantil en la Universidad de Costa Rica. Instituto de Investigaciones y Mejoramiento Educativo. Guatemala, 1962.

16. Friis R, Sellers T. Epidemiology for Public Health Practice. Maryland, United States of America: An Aspen Publication, 1999; 506 pp.

17. Montesinos E, Alberto E. Las necesidades y motivos como factores fundamentales de las estudiantes de enfermería. Rev Cub Enferm 1988; 4 (1/2): 79-88.

18. Álvarez Z, Ruz L. La formación vocacional y orientación profesional a través de los círculos de interés científicotécnicos. Rev Cuba Enferm 1987; 3(3): 221-37.

19. Gonzales LF. Estudio de los factores socioeconómicos en la elección profesional. Monografía para optar el grado de Bachiller en Ciencias de la Educación, Universidad Nacional de Educación Enrique Guzman y Valle. Chosica (Perú), 1977.

20. Loarte A. Cambios en la modernidad y su impacto en las organizaciones. Caso práctico: Universidad Nacional Pública. Tesis para optar el título de Licenciado en Administración. Universidad Nacional de Educación Enrique Guzmán y Valle. Lima (Perú), 1998.

21. Varela L. Factores causantes de la elección profesional. Tesis de Bachiller en Educación. Universidad Nacional "Enrique Guzmán y Valle”. Lima (Perú), 1977.

22. Rodríguez V. Importancia de la orientación vocacional y profesional en la elección de carreras. Experiencia en la Academia Preuniversitaria Pitágoras. Tesis de Licenciada en Psicología. Universidad Nacional Federico Villarreal, Facultad de Psicología. Lima (Perú), 1996.

23. Minchola A. Factores socio-económicos influyentes en el rendimiento de los estudiantes de enfermería. Trabajo de Habilitación para Ascenso en la Docencia, Departamento de Medicina Preventiva y Salud Pública. Universidad Nacional de Trujillo. Trujillo, 1976. 
24. Tipismana O. Correlación estrés psicológico y rendimiento académico en estudiantes de enfermería del servicio de sanidad de la Policía Nacional. Tesis de Licenciada en Psicología. Universidad Nacional Federico Villarreal, Facultad de Psicología. Lima (Perú), 1992.

25. Morante L. Atribuciones de éxito y fracaso académcio y su relación con el rendimiento académico de un grupo de estudiantes universitarios de estomatología. Tesis para optar título de Licenciado en Psicología. Universidad Peruana Cayetano Heredia. Lima (Perú), 1998.

26. Scarinci IC, Utyama IK, Guariente de Menezes MH, Ohnishi M, Mussi NM. Apoio psicológico: uma necessidade dos alunos de enfermagem. Semina 1989; 10 (2): 98-103.

27. Miljánovic M. Frustración y rendimiento académico en estudiantes universitarios. Alma Mater 1993: 5: 99-104.
28. Jondec D, Amaya N. Prevalencia de síndrome depresivo en las alumnas de enfermería. Tesis de Bachiller en Enfermería. Universidad Nacional de Trujillo, 1985.

29. Hornung M. Lucha antituberculosa universitaria. Tesis de Bachiller en Medicina. Facultad de Medicina, Universidad Nacional Mayor de San Marcos. Lima (Perú), 1942.

30. Sánchez JJ. Deserción: causas académicas, socioeconómicas y culturales en la Escuela de Ingeniería Mecánica de la UNT. Tesis para optar el grado de Maestro en Tecnología Educativa. Universidad Nacional de Trujillo. Escuela de Postgrado de la Facultad de Educación. Trujillo (Perú), 1993.

31. Lens W. El rol de la perspectiva de tiempo futuro en la motivación estudiantil. Persona 1998; 1: 67-94.

32. Montes I, Palomino E. El ingreso a las universidades peruanas. Lima (Perú): Edit. Talleres Gráficos de Litho Print, Consorcio Centros educativos católicos del Perú. 1993; 71 pp.

\section{JORNADAS DE INVESTIGACIÓN EN SALUD 2002 FE DE ERRATAS}

* Estudio comparativo de marcadores serológicos de hepatitis B (HBsAg y HBcAb) en donantes de zonas endémicas del Perú

Rivera J.

Por: Fuentes-Rivera J.

* Seroprevalencia de anticuerpos de malaria en donantes de sangre de zonas endémicas

Rivera J.

Por: Fuentes-Rivera J.

* Prevalencia de hepatitis $\mathrm{C}$ en donantes de sangre de la costa y selva peruanas

Rivera J.

Por: Fuentes-Rivera J.

* Perfil del donante de sangre en el Perú

Rivera J.

Por: Fuentes-Rivera J.

* Traumatismo cardiopericárdico penetrante. Experiencia en el Hospital Nacional Dos de Mayo de 6 casos durante 1999 - 2002

Yarihuamán R, Montesinos E, Rodríguez J, Rojas L, Castillo L.

Por: Rafael A, Montesinos E, Peralta J, Rojas L, Castillo L.

* Purificación de antígenos de Paragonimus mexicanus para el inmunodiagnóstico de la paragonimiosis humana

Cornejo W, Sevilla C, Betalleluz P, Huiza A.

Por: Cornejo W, Sevilla C, Alva P, Huiza A. 